\title{
Energy efficient clustering method for wireless sensor network
}

\author{
Chaitra H.V ${ }^{1}$, Dr. Ravikumar G.K ${ }^{2}$ \\ ${ }^{1} \mathrm{NMIT}$, VTU, Bangalore, India \\ ${ }^{2}$ VTU, Belgaum, India
}

\begin{tabular}{l}
\hline \hline Article Info \\
\hline Article history: \\
Received Sep 28, 2018 \\
Revised Nov 11, 2018 \\
Accepted Dec 21, 2018 \\
\hline
\end{tabular}

\section{Keywords:}

Clustering

Evolutionary computing WSN

\begin{abstract}
Wireless sensor has attained wide interest across various industries due to availability of low cost sensor devices. Preserving battery/energy of these sensor devices are most desired. Recently, many approaches has been presented to improve lifetime of sensor networks adopting clustering technique. Cluster head selection play an important factor in improving lifetime of cluster based network. For improving cluster head selection multiobjective function are presented in recent time by adopting evolutionary computing and metaheuristic algorithm. However, the existing model incurs computation overhead due to NP-Hard problem and connectivity issues is not considered. Thus affecting network performance. To address the research issues, this work present a novel Multi-objective imperialist competitive algorithm (MOICA) for cluster head selection and routing optimization. Experiment are conducted to evaluate the performance of MOICA over LEACH in term of lifetime performance considering first sensor node death and $75 \%$ sensor node death. The outcome shows an average lifetime performance improvement of $56.32 \%$ in case of first sensor node death and $53.61 \%$ in case of $75 \%$ sensor node death.Therefore, MOICA achieves significance improvement over LEACH based protocols.
\end{abstract}

Copyright (C) 2019 Institute of Advanced Engineering and Science. All rights reserved.

\section{Corresponding Author:}

Chaitra H. V, NMIT, VTU, Bangalore, India.

Email: chaitrahvgowda2005@rediffmail.com

\section{INTRODUCTION}

WSN is composed of large number of low cost and tiny sensor devices. The sensor devices are powered by battery and are deployed remotely. The WSN is adopted in real time sensing and processing of data such as pressure, humidity, temperature, gas etc. WSN is been adopted in various real time application such as disaster management, surveillance, forest fire detection etc. and has been current hot research in various industries and organization. The sensor devices are deployed in hazardous locations where recharging of battery or replacing it is not a viable option, where human monitoring involves high risk. The sensor device has constraint of limited computing capability, radio connectivity and batter capacity which makes sensor nodes fails some time. The sensor nodes are composed of event driven or time driven. In event driven the sensor device sense data when there is a change in event occurs. In time driven the sensor device sense data based on predefined time. In both case the energy of battery is decreased exponentially. The sensed data are either transmitted to neighbouring node or to the sink/base station. Sometime the same data are transmitted to base station as a result affecting energy efficiency of senor network. To overcome the redundancy issue, data aggregation and sensor fusion have been adopted in [1] to make the network energy efficient.

To provide real time access [2] of data to sensor data for efficient industries applications, accurate gathering and processing of data is required [3]. But gathering these data possess a challenge. A solution to this is presented in [4]. To provision efficient data gathering, energy conservation scheme [5] is required. Energy conservation routing model adopting clustering technique such as LEACH, HEED and TEEN has been presented in literature [6]. However these models induce energy overhead for cluster head and are not suitable 
for large network. Since direct transmission of packet via cluster head toward base station is not a feasible strategy for large network. In [7] and [8] presented a distributed algorithm to improve the energy efficiency of sensor network. In [9] presented a routing algorithm for hop node selection for cluster network to improve life time of sensor networks. However their model induces high communication overhead among hop nodes and cluster devices due to channel contention and optimizing it is NP-deterministic.

Evolutionary computing model is adopted to solve energy efficiency and cluster head selection optimization issue (.i.e. NP-hard problem) in WSN. In [10] carried out extensive survey of meta-heuristic optimization algorithm such as Genetic Algorithm (GA), Particle Swarm Optimization (PSO) and Imperialist Competitive Algorithm (ICA). These algorithms are adopted to solve various routing and clustering problem in WSN to enhance lifetime of sensor networks. The outcome presented in [11] and [12] shows that ICA perform better than GA and PSO. They highlighted that GA can solve global optimization problems. However, they are easily trapped in a local optimal solution and their executions are time-consuming [13]. PSO has numerous complications, such as easily falling into the local optimum and premature convergence [14]. The ICA model presented in [12] adopted PSO to prevent colonies from moving beyond search space. However their model is not efficient for multi-objective problems. To overcome the research challenges, this work present a Multi-objective ICA (MOICA) optimization technique for cluster networks to improve lifetime of wireless sensor networks.

The model first adopt hop based transmission for inter cluster communication in order to prevent preserve energy of cluster head device. Secondly, for every cluster head there is corresponding Hop/Cluster head device. This aids in reducing the energy consumption of cluster head device and also channel contention problem in selecting hop device is eliminated. Then selection of hop device is carried out based on distance and energy remaining. Then fitness function is generated for selecting cluster head and hop device. The selection hop and cluster devices is formulated as Np-hard problem. An improved ICA is presented to obtain an ideal solution. The lifetime of sensor networks are generally defined by number of round. However, death of one node may affect the lifetime of sensor networks due to loss of connectivity. No prior works have carried experiment outcomes interm of first node death and total node death for varied network density. This work carried out experiment evaluation for all these lifetime parameter shows significant performance improvement over state of art technique.

The paper organization is as follows: The literature survey is presented in section two. The proposed models are presented in Section three. The simulation results and the experimental study are presented in the penultimate four. The concluding remark and future work is discussed in the last section.

\section{LITTERATURE SURVEY}

Recently several methodologies has been presented to enhance the performance of wireless sensor network which are surveyed here. In [9] presented an energy efficient routing model to address the challenges in gathering real time data generated by Big Data industries. The Big Data algorithm (BDEG) is presented for data collection in wireless sensor network. They adopted cluster based routing model. Clustering communication is established based on residual energy and radio signal strength indicator (RSSI) of sensor device. Their experimental outcome shows the BDEG model is stable in terms of data transmission time and network lifetime because of load balancing technique adopted by them. However, the total distance in multihop communication is much higher than the single hop data transmission as a result it increase the energy consumption of individual sensor device thus may result in loss of connectivity. In [15], identified the relation between routing and clustering and presented a joint clustering routing (JCR) scheme for reliable and efficient design. They presented an optimization technique to address the unbalanced cluster head selection in multihop network. The outcome shows the model improves the connectivity of network. In [16] hierarchical based energy efficient reliable routing protocol for WSN. In their design, depending on network topology the data transmission from cluster head and base station is carried directly or through multi-hop fashion. Outcome shows significant performance over state-of-art technique. In [17] presented a type 2 fuzzy based clustering model which is an enhancement of the fuzzy based clustering model presented in [18]. The model is expected to handle uncertain level decision, thus enhancing lifetime performance. However, connectivity issue is not addressed here. To address the lifetime and connectivity issue [19] presented a multi-objective optimization for cluster head selection. Their model considered energy, packet loss rate and link quality as a multi-objective parameter. To minimize energy consumption of cluster head, relay nodes are selected for performing multihop transmission. The result shows performance improvement in term of lifetime and throughput. Extensive research survey carried out in [10] shows that meta-heuristic algorithm such as PSO, GA, ICA is efficient for optimizing cluster selection. However, the model presented in [12] shows the existing ICA model performs better than its counterpart PSO and GA. However, their model does not consider multi-objective parameter for cluster head selection and they suffers from $\mathrm{Np}$-hard problems. 
Extensive research survey carried out shows various methods have be applied to solve the energy efficiency and life time requirement of sensor network. The survey shows adopting multi-objective function aid in improving network lifetime. However, for better cluster head selection metaheuristic algorithm is adopted. However, the Np-hard issue needs to be taken care of. Most of exiting metaheuristic algorithm are designed single objective and connectivity issues are neglected. To address the research challenge in next we present our proposed optimized multi-objective imperialist clustering algorithm for WSN.

\section{PROPOSED MULTI-OBJECTIVE IMPERIALIST COMPETITIVE ALGORITHM}

Here the author proposes an energy efficient design for cluster head selection based on connectivity and Energy efficiency. The optimization for energy efficient protocol is done by applying Imperialist Competitive Algorithm (ICA). The transmission in cluster network is classified into following as inter clustering and intra clustering transmission which is shown in Figure 1.

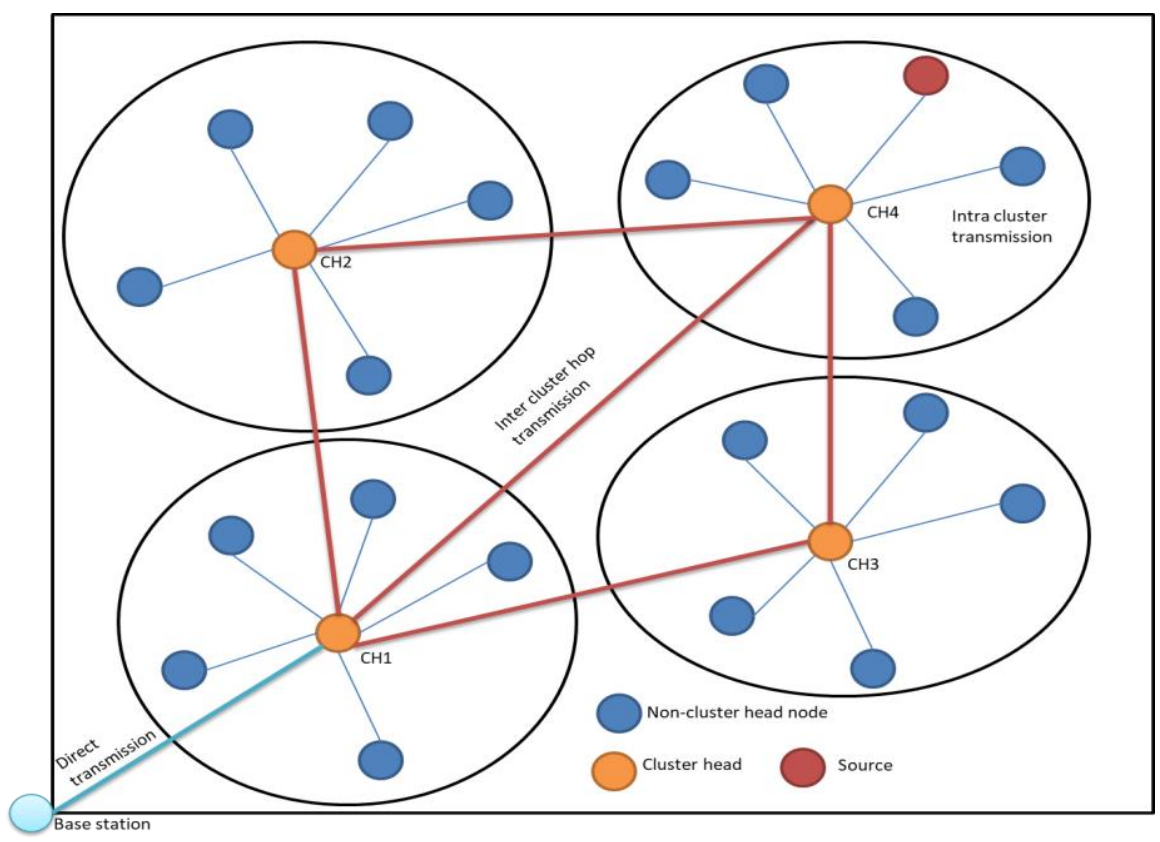

Figure 1. Architecture of proposed model

Here we present a clustering model using Imperialist Competitive Algorithm (ICA). The sensor devices are classified as normal sensor devices and cluster head devices. The proposed model consist of two stages, setup and transmission stage. In setup stage, the cluster member, cluster head, route among the clusters and the base station are determined. In transmission stage, the cluster head collects and aggregate the data from its member and transfer this data to its best optimized cluster head toward base station.

Let consider that there are $K$ sensor device which are arbitrarily deployed in a network area, which are further partitioned into $k$ clusters. The cluster head sets $\mathcal{D}$ is defined as $\mathcal{D}=\left\{\mathcal{D}_{1}, \mathcal{D}_{2}, \mathcal{D}_{3}, \ldots, \mathcal{D}_{y}, \ldots, \mathcal{D}_{k}\right\}$, and set of non-cluster devices as $\widetilde{\mathcal{D}}$. The cluster head collect the sensory information from member device, aggregate the data of intra cluster member and routes aggregated data to the best optimized cluster head. For selecting the cluster head, the remaining energy and position of sensor devices are considered. The base station selects the node with maximum energy and has better connectivity as a cluster head and each cluster have equal distribution of member device. This process is consider as an optimization problem which can be expressed as follows

$$
O_{\mathcal{D}}=\gamma * T_{h}^{\mathcal{D}}+(1-\gamma) * T_{m}^{\mathcal{D}}
$$

where $\gamma$ is the cost optimization constant, $T_{h}^{\mathcal{D}}$ is the ratio of cluster head mean remaining energy with respect to member device, $T_{m}^{\mathcal{D}}$ is the ratio of mean distance among non-cluster head and the sink to the mean distance among the cluster head and the base station. The optimization problem in (1), comprises of two things, 
where $\gamma$ is a constant which depicts the impact of $T_{h}^{\mathcal{D}}$ and $T_{m}^{\mathcal{D}}$ in computing cost optimization param $O_{\mathcal{D}}$. The mean remaining energy of current round is computed as follows,

$$
\begin{aligned}
& T_{h}^{\mathcal{D}}=\vec{L}_{\mathcal{D}} / \vec{L}_{\widetilde{\mathcal{D}}} \\
& T_{h}^{\mathcal{D}}={ }^{\sum_{\forall s_{y} \in \mathcal{D}} L_{\mathcal{D}}^{i}(y) /|\mathcal{D}|} / \sum_{\forall s_{x} \in \widetilde{\mathcal{D}}} L_{\widetilde{\mathcal{D}}}^{i}(x) /|\widetilde{\mathcal{D}}|
\end{aligned}
$$

where $\vec{L}_{\widetilde{D}}$ is the mean remaining energy of cluster member device and $\vec{L}_{\mathcal{D}}$ is the mean remaining energy of cluster head, $|\widetilde{\mathcal{D}}|$ and $|\mathcal{D}|$ denotes the number of cluster member device and number cluster head devices respectively. $s_{y}$ denotes the node $y \in$ cluster head. The sensor device with maximum $T_{h}^{\mathcal{D}}$ is selected as cluster head.

The $T_{m}^{\mathcal{D}}$ is computed as follows,

$$
\begin{aligned}
T_{m}^{\mathcal{D}} & =\vec{E}_{\widetilde{\mathcal{D}}} / \vec{E}_{\mathcal{D}} \\
T_{m}^{\mathcal{D}} & =\sum_{\forall s_{x} \in \widetilde{\mathcal{D}}} e\left(s_{x}, \mathcal{S}\right) /|\widetilde{\mathcal{D}}| / \sum_{\forall s_{x} \in \mathcal{D}} e\left(s_{x}, \mathcal{S}\right) /|\mathcal{D}|
\end{aligned}
$$

where $e\left(s_{x}, \mathcal{S}\right)$ represent the distance among base station $\mathcal{S}$ and node $x$. To improve the energy of sensor network, the objective function $T_{m}^{\mathcal{D}}$ can be maximized to optimize better cluster formation and cluster head selection.

To reduce the energy consumption of cluster device for inter cluster transmission, hop nodes (Cluster head) are selected to transmit data. The hop devices are selected based on following condition. Firstly, the cluster head and hop device must possess higher energy than normal sensor device. Secondly, the hop device should possess better location between base station and cluster head, in order to minimize energy consumption. To reduce the computation cost among cluster head and hop nodes, the cluster selects the neighbouring cluster head as its hop device.

The set of inter cluster hop device can be represented as $\mathbb{D}=\left\{\mathbb{D}_{1}, \mathbb{D}_{2}, \mathbb{D}_{3}, \ldots, \mathbb{D}_{u}, \ldots, \mathbb{D}_{v}\right\}$ and set of normal sensor devices as $\mathbb{S}$. The cost function for selection hop device for inter cluster transmission is given as follows

$$
O_{\mathbb{D}}=\varphi * T_{h}^{\mathbb{D}}+(1-\varphi) * T_{m}^{\mathbb{D}}
$$

where $T_{h}^{\mathbb{D}}$ is ratio of inter cluster hop devices remaining energy over normal sensor devices which is defined as follows

$$
\begin{aligned}
& T_{h}^{\mathbb{D}}=\vec{L}_{\mathbb{D}} / \vec{L}_{\mathbb{S}} \\
& T_{h}^{\mathbb{D}}=\sum_{\forall s_{u} \in \mathbb{D}} L_{\mathbb{D}}^{i}(u) /|\mathbb{D}| / \sum_{\forall s_{q} \in \mathbb{S}} L_{\mathbb{S}}^{i}(q) /|\mathbb{S}|
\end{aligned}
$$

where $|\mathbb{S}|$ and $|\mathbb{D}|$ denote the number of normal sensor devices and inter cluster hop devices respectively, $\vec{L}_{\mathbb{D}}$ is the mean reaming energy of inter cluster hop devices. The device with higher energy is selected as the inter cluster hop device by maximizing $T_{h}^{\mathbb{D}}$. Similarly the $T_{m}^{\mathbb{D}}$ can be expressed as follows,

$$
\begin{aligned}
& T_{m}^{\mathbb{D}}=\vec{Z}_{\mathbb{S}} / \vec{Z}_{\mathbb{D}} \\
& T_{m}^{\mathbb{D}}={ }^{\sum_{\forall s_{q} \in \mathbb{S}}}\left\{e\left(s_{q}, \mathcal{S}\right)+e\left(s_{q}, \mathcal{D}_{y}\right)\right\} / \mathbb{S} / \sum_{\forall s_{u} \in \mathbb{D}}\left\{e\left(\mathbb{D}_{u}, \mathcal{S}\right)+e\left(\mathbb{D}_{u}, \mathcal{D}_{y}\right)\right\} / \mathbb{D}
\end{aligned}
$$


For selection of cluster head $\mathcal{D}_{y}$ and its corresponding inter cluster hop device $\mathbb{D}_{u}$, the location of base station $\mathcal{S}$ and cluster head $\mathcal{D}_{y}$ is considered. The transmission cost among cluster head and inter cluster hop device can be reduced by maximizing $T_{m}^{\mathbb{D}}$.

The optimization problem of cluster head selection is solved by applying the modified ICA.

Firstly initialize the optimization problem and parameters, initialize a set of countries and the size of countries is expressed as $M$, each countries $i$ has a position vector $g_{a}=\left[g_{a 1}, g_{a 2}, g_{a 3}, \ldots, g_{a j}\right]$ and velocity vector $w_{a}=\left[w_{a 1}, w_{a 2}, w_{a 3}, \ldots, w_{a d}\right]$ is used to specify the current state, where $a$ is a positive param indexing the countries in a colony and $j$ depicts the problem dimension.

Secondly we compute the fitness of each country, each country compute its fitness function based on (1) and (6). During this phase each country maintains local best solution $R_{a}=\left[r_{a 1}, r_{a 2}, r_{a 3}, \ldots, l_{l j}\right]$ by itself and global best solution $R_{l}=\left[r_{l 1}, r_{l 2}, r_{l 3}, \ldots, l_{a j}\right]$ achieved by any country in a colony. Then it computes and finds the global and local best position based on which imperialist are added.

Thirdly updating position and velocity, in each round there is a change in velocity of each country towards local best and global best positions. The position of countries is updated as follows

$$
g_{a b}^{\mathbb{t}+1}=g_{a b}^{\mathbb{t}}+w_{a b}^{\mathbb{t}+1}
$$

The velocity of countries is updated as follows

$$
w_{a b}^{\mathbb{t}+1}=\mathbb{v} w_{a b}^{\mathbb{t}}+\mathbb{U n}_{1} \mathbb{Z}_{1}\left(r_{a b}^{\mathbb{t}}-g_{a b}^{\mathbb{t}}\right)+\mathbb{U n}_{2} \mathbb{Z}_{2}\left(r_{l b}^{\mathbb{t}}-g_{l b}^{\mathbb{t}}\right)
$$

where the notation of $g_{a b}, r_{a b}$ and $r_{a b}$ is similar to $w_{a b} . w_{a b}$ is the bth dimension of ath countries velocity and it is generally limited to closed interval of $\left[w_{\downarrow}, w_{\uparrow}\right]$ to prevent colonies from moving beyond the search space boundary conditions. The acceleration param $\mathrm{un}_{1}$ and $\mathrm{un}_{2}$ are controlled based on evolutionary states. Coefficient $\mathbb{Z}_{1}$ and $\mathbb{Z}_{2}$ are arbitrarily generated param between zero to one for $j$ th dimension and $\mathbb{v}$ is the inertia weight. The weights $\mathbb{V}$ play a critical part in controlling influence of velocity of a country of present one. This is done to bring tradeoff between global and local search (i.e. large and small inertial weight update respectively).

The updation of weight is modified to prevent the proposed optimization model in getting stuck in local optima which is as follows

$$
\mathbb{V}=\left(\frac{\mathbb{I}_{\uparrow}-\mathbb{I}_{\mathcal{C}}}{\mathbb{I}_{\uparrow}}\right) *\left(\mathbb{V}_{\uparrow}-\mathbb{V}_{\downarrow}\right)+\mathbb{V}_{\downarrow}
$$

where $\mathbb{I}_{\uparrow}$ is the maximum amount iteration permitted, $\mathbb{I}_{\mathcal{C}}$ is the present iteration, $\mathbb{V}_{\downarrow}$ is the minimum inertial weight and $\mathbb{V}_{\uparrow}$ is the maximum inertial weight. The present finest solution is chosen after termination statement is met. This is the ideal strategy for optimization is computed.

The data aggregated by cluster is as follows: $o^{t h}$ sensor node transmits the data of $b_{o}$ bits to its cluster head. The total data $\mathcal{B}_{h}$ bits gathered by cluster head are computed has follows

$$
\mathcal{B}_{h}=\sum_{o=1}^{h} b_{o}
$$

where $h$ is the number of members in a cluster.

The energy consumed to sense data, transmit, receive data and sleep schedule is computed as follows

$$
\begin{aligned}
& L_{\mathbb{S}}=\left(1-l_{\text {sleep }}\right)\left[L_{\text {src }}(\mathbb{q}, \mathbb{e})+L_{\text {dst }}(\mathbb{q})\right]+l_{\text {sleep }} L_{\text {sleep }} \\
& L_{\mathbb{S}}=\left(1-l_{\text {sleep }}\right)\left(\mathbb{q} L_{\text {elec }}+\mathbb{q} L_{\mathbb{F}} * \frac{\mathbb{A}^{2}}{2 n \pi}+\mathbb{q} L_{\text {elec }}\right)+l_{\text {sleep }} L_{\text {sleep }}
\end{aligned}
$$

where $l_{\text {sleep }}$ is likelihood of sleep schedule of sensor devices, $L_{\text {sleep }}$ is the energy dissipation of sensor node in sleep mode. Each cluster head will receive data from member of sensor device. The cluster head then aggregates and transmit data to inter cluster hop device. The inter cluster hop device are reasonably close to cluster head, hence free space model is used.to compute energy dissipation of cluster head, which is as follows

$$
L_{\mathcal{D}}=L_{s r c}(\mathfrak{q}, \mathbb{e})+\left(\frac{\mathbb{H}}{\mathbb{h}}-2\right) L_{d s t}(\mathfrak{q})+\frac{\mathbb{H}}{\mathbb{h}} \mathfrak{q} \mathcal{B}_{h}
$$




$$
\begin{aligned}
& L_{\mathcal{D}}=\mathbb{q} L_{\text {elec }}+\mathfrak{q} L_{\mathbb{F}} \frac{\mathbb{A}^{2}}{2 n \pi}+\left(\frac{\mathbb{H}}{n}-2\right) \mathbb{q} E_{\text {elec }}+\frac{\mathbb{H}}{n} \mathfrak{q} \mathcal{B}_{h} \\
& L_{\mathbb{D}}=\left(1-l_{\text {sleep }}\right)\left[L_{\text {src }}(\mathfrak{q}, \mathbb{e})+L_{\text {dst }}(\mathfrak{q})\right]+l_{\text {sleep }} L_{\text {sleep }}
\end{aligned}
$$

where $\mathcal{B}_{h}$ is the energy consumption per bit due to data aggregation. The aggregated data is then sent to inter cluster hop device and it is sent to base station. The inter cluster hop device is generally positioned far away from base station as a result multi-path model is used. The energy dissipation of inter cluster hop device is computed as follows

$$
L_{\mathbb{D}}=\left(1-l_{\text {sleep }}\right)\left(\mathbb{q} L_{\text {elec }}+\mathbb{q} L_{\mathbb{M}} \mathbb{e}_{\mathcal{S}}^{4}+q \mathbb{1} L_{\text {elec }}\right)+l_{\text {sleep }} L_{\text {sleep }}
$$

where $\mathbb{e}_{S}^{4}$ is the distance between inter cluster hop device and the base station. Therefore the energy consumption for both inter and intra cluster routing is given as follows

$$
L_{\mathbb{T}}=L_{\mathcal{D}}+L_{\mathbb{D}}+\left(\frac{\mathbb{H}}{\mathbb{h}}-2\right) L_{\mathbb{S}}
$$

Therefore the total energy consumption of network can be obtained as

$$
L_{\mathcal{T}}=\mathbb{h} L_{\mathbb{T}}
$$

Therefore the energy dissipation of a network depends on the position of base station, number of sensor device and size of network topology.

\section{SIMULATION RESULTS AND ANALYSIS}

The system environment used is windows 10 enterprises operating system, 64-bit Quad core processor, 2GB NVDIA CUDA Dedicated Graphic card, with 16GB of RAM. We have used sensoria simulator [19] which is designed using dot net framework 4.0 and C\# as a programming language. We have conducted simulation study to evaluate network lifetime performance considering first node death and $75 \%$ node death and compared our proposed MOICA model with existing $L E A C H$ based protocol and we have varied node size by 400,600 and 800 and conducted simulation study and the simulation parameter used for experimental analysis is shown in Table 1.

Table 1. Simulation Parameter Considered

\begin{tabular}{ll}
\hline Network Parameter & Value \\
\hline Network Size & $40 \mathrm{~m} * 50 \mathrm{~m}$ \\
Number of sensor nodes & $400,600,800$ \\
Number of Base station & 1 \\
Initial energy of sensor nodes & $0.2 \mathrm{~J}$ \\
Radio energy dissipation & $50 \mathrm{nj} / \mathrm{bit}$ \\
Data packets length & $2000 \mathrm{bits}$ \\
Transmission speed & $200 \mathrm{bit} / \mathrm{s}$ \\
Bandwidth & $10000 \mathrm{bit} / \mathrm{s}$ \\
Idle energy consumption (Eelec) & $50 \mathrm{nj} / \mathrm{bit}$ \\
Data packet processing delay & $0.1 \mathrm{~ms}$ \\
Amplification energy (Emp) & $100 \mathrm{pJ} / \mathrm{bit} / \mathrm{m} 2$ \\
\hline
\end{tabular}

\subsection{Network Lifetime Performance Considering First Sensor Node Death}

In Figure 2 network lifetime is presented for the 400 nodes after death of first sensor for both proposed MOICA and existing LEACH. For LEACH first node death observed after 183 rounds while for MOICA method first sensor node death observed after 328 rounds. In Figure 3 network lifetime is presented for the 600 nodes after death of first sensor for both MOICA and existing system. For LEACH first node death observed after 138 rounds while for MOICA method first sensor node death observed after 282 rounds. In Figure 3 network lifetime is presented for the 800 nodes after death of first sensor for both MOICA and existing system. For LEACH first node death observed after 74 rounds while for MOICA method first sensor node death observed after 280 rounds. It is observed from Figures 2, 3 and 4 that MOICA improves lifetime performance 
by $44.21 \%, 51.17 \%$ and $73.58 \%$ considering 400,600 and 800 sensor nodes respectively over LEACH protocol. An average lifetime improvement of $56.32 \%$ is achieved by MOICA over LEACH protocol.

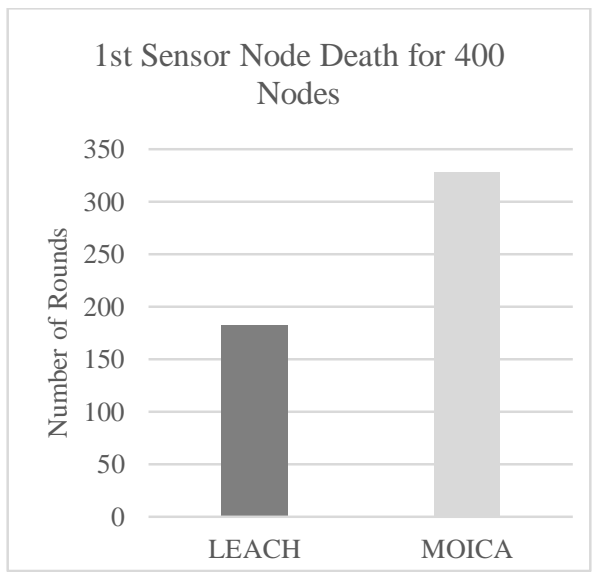

Figure 2. Network lifetime performance for 400 sensor node considering first sensor node death

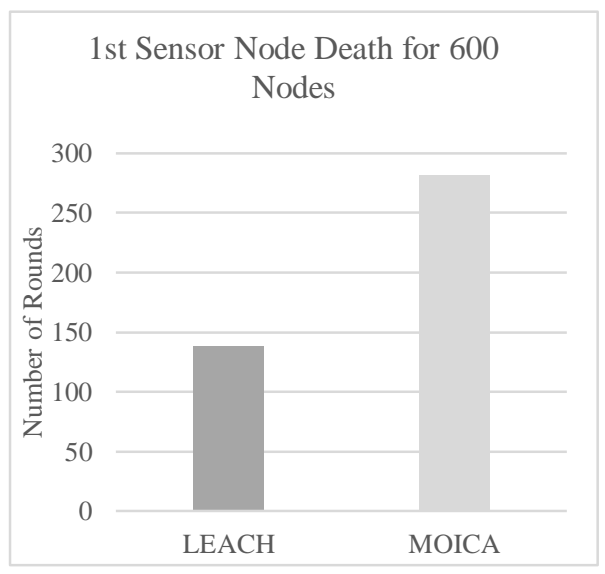

Figure 3. Network lifetime performance for 600 sensor node considering first sensor node death

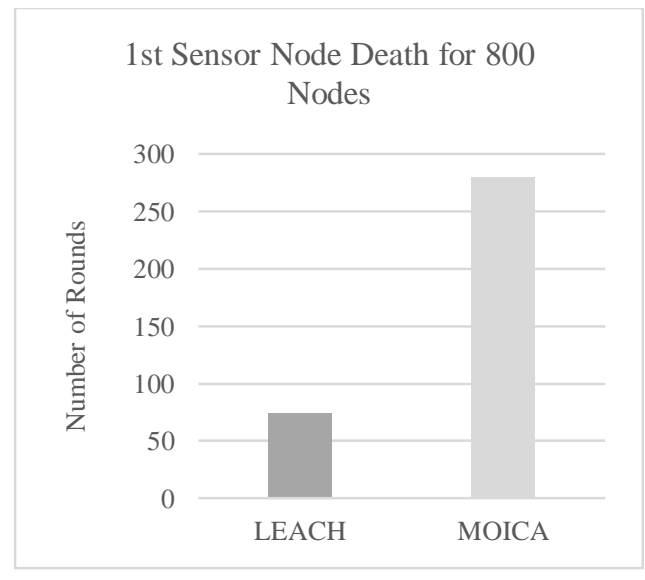

Figure 4. Network lifetime performance for 800 sensor node considering first sensor node death

Network lifetime performance considering $75 \%$ sensor node death:

In Figure 5 network lifetime is presented for the 400 nodes after death of $75 \%$ sensor for both proposed MOICA and existing LEACH system. For LEACH 75\% node death observed after 653 rounds while for proposed method $75 \%$ sensor node death observed after 886 rounds. In Figure 6 network lifetime is presented for the 600 nodes after death of $75 \%$ sensor for both MOICA and LEACH. For LEACH 75\% node death observed after 367 rounds while for MOICA method 75\% sensor node death observed after 963 rounds. In Figure 7 network lifetime is presented for the 800 nodes after death of $75 \%$ sensor for both MOICA and LEACH. For LEACH 75\% node death observed after 269 rounds while for MOICA method $75 \%$ sensor node death observed after 1049 rounds. It is observed from Figures 5, 6 and 7 that MOICA improves lifetime performance by $24.6 \%, 61.89 \%$ and $74.36 \%$ considering 400,600 and 800 sensor nodes respectively over LEACH protocol. An average lifetime improvement of $53.61 \%$ is achieved by MOICA over LEACH protocol. 


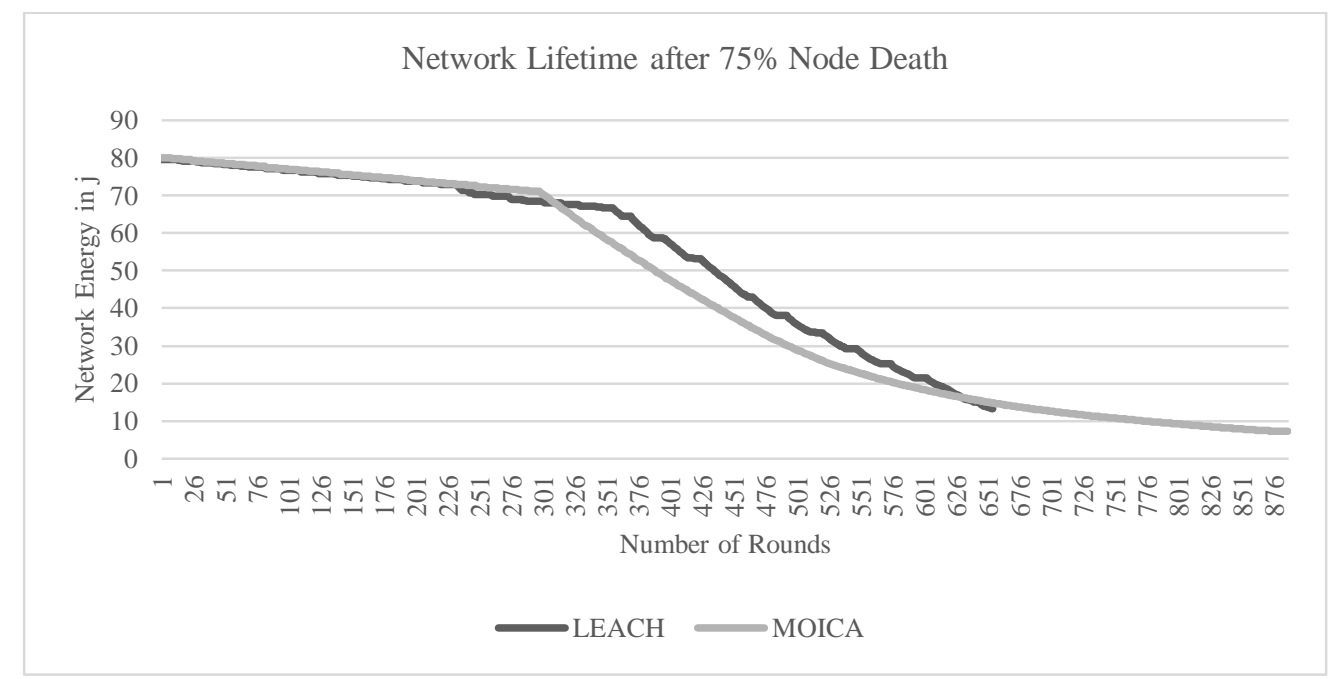

Figure 5. Network lifetime performance for 400 sensor nodes considering $75 \%$ sensor node death

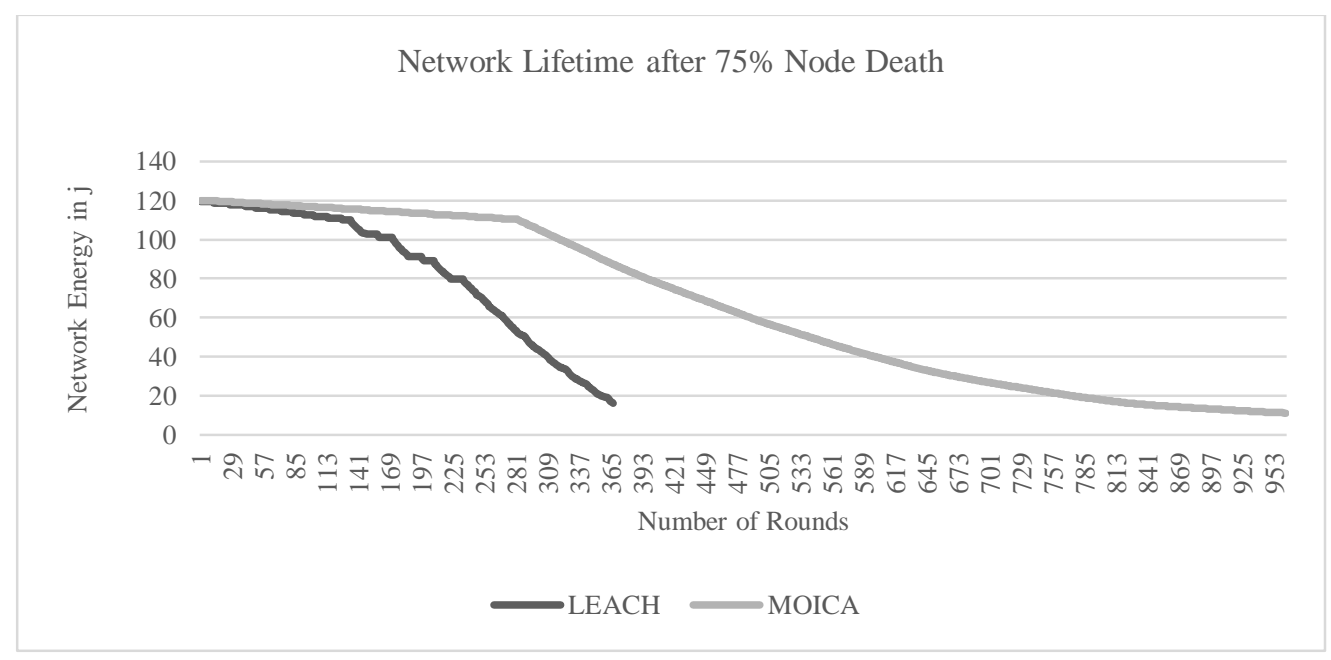

Figure 6. Network lifetime performance for 600 sensor nodes considering $75 \%$ sensor node death

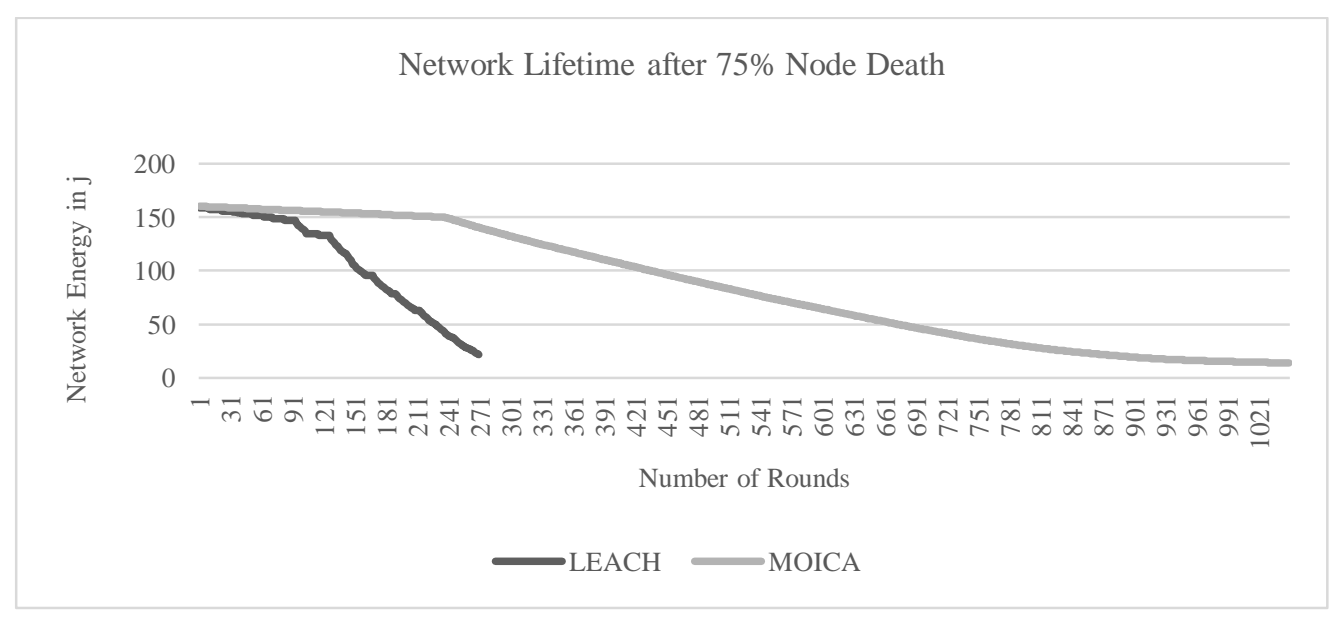

Figure 7. Network lifetime performance for 800 sensor nodes considering $75 \%$ sensor node death 


\subsection{Comparsion of Proposed technique with state-of-art techniques}

Table 2 shows performance comparison of proposed system (PS) and existing protocols average lifetime achievement over LEACH [20] protocol. The model presented in [21] considered performance evaluation over LEACH considered a maximum of $\mathbf{2 0 0}$ sensor nodes and attained a lifetime improvement of 24.0\% over LEACH, [22] considered a maximum of $\mathbf{4 0}$ sensor nodes and achieved a lifetime improvement of 25.0\% over LEACH, [23] considered a maximum of $\mathbf{1 0 0}$ sensor nodes and attained an lifetime improvement of $\mathbf{1 5 . 0 \%}$ over LEACH, [24] considered a maximum of $\mathbf{1 0 0}$ sensor nodes and achieved a lifetime improvement of $\mathbf{1 5 . 8 2 \%}$ over LEACH, and [25] considered a maximum of $\mathbf{4 0}$ sensor nodes and achieved a lifetime improvement of $\mathbf{4 7 . 3 3 \%}$ over LEACH. The state-of-art considered performance evaluation considering $\mathbf{4 0}$ to 200 sensor devices which is very small considering the future application needs.

Our work considered performance evaluation considering 400 to 1000 sensor devices and the average lifetime performance outcome is measure in term of percentage and is tabulated in Table 2. The outcome shows the proposed system attain an average throughput performance improvement of $56.96 \%$. The overall outcome shows that the PS model achieved significant network lifetime performance improvement over state-of-art model [21], [22], and [23] considering network lifetime.

Table 2. Performance comparison of proposed system (PS) and existing protocols average lifetime achievement over LEACH

\begin{tabular}{cc}
\hline Algorithm & $\begin{array}{r}\text { Average lifetime \% improvement } \\
\text { over LEACH (ES) [20] }\end{array}$ \\
\hline REAC-IN [21] & $24.0 \%$ \\
LEACH-Fuzzy [22] & $25.0 \%$ \\
$E^{2} R^{2}[23]$ & $15.0 \%$ \\
TSEP[24] & $15.82 \%$ \\
DCH-GA[25] & $47.33 \%$ \\
Proposed system (PS) & $56.96 \%$ \\
\hline
\end{tabular}

\section{CONCLUSION}

Clustering technique play an important factor in enhancing the lifetime of sensor networks. However, improper cluster selection techniques will lead to performance degradation. For better cluster selection evolutionary computing is adopted by existing approaches. However, they suffers NP-Hardness problem and connectivity issues is neglected. To overcome research challenges, this work presented Multi-objective imperialist competitive algorithm (MOICA) for cluster head selection and routing optimization. Experiment are conducted to evaluate the performance of MOICA over LEACH in term of lifetime performance considering first sensor node death and 75\% sensor node death. The outcome shows an average lifetime performance improvement of $56.32 \%$ and $53.61 \%$ is achieved by MOICA over LEACH based protocols. The outcome of proposed model achieved show that it achieves significance performance improvement when compared to $\mathrm{E}^{\wedge} 2 \mathrm{R}^{\wedge} 2$ (energy-Efficiency and Reliable Routing) proposed by H. K. Deva Sharma et al. In future this work would consider evaluating performance analysis consider varied network parameters. The performance achieved of our model interm of energy efficiency will aid in providing security to these sensor devices. Our future work will embed security to sensor networks and evaluate the performance and highlight its significance and energy overhead induced in providing security.

\section{REFERENCES}

[1] R. Sathish Kumar, R.Logeswari, N.Anitha Devi, S.DivyaBharathy, "Efficient Clustering using ECATCH Algorithm to Extend Network Lifetime in Wireless Sensor Networks", International Journal of Engineering Trends and Technology (IJETT) - Volume-45, Number-9, 2017.

[2] Bellavista, P., Cardone, G., Corradi, A., and Foschini, L., "Convergence of MANET and WSN in IoT urban scenarios”, IEEE Sensors Journal, 13(10), 3558-3567, 2013.

[3] Z. Kremljak and C. Kafol, "Types of risk in a system engineering environment and software tools for risk analysis", Proc. Eng., vol. 69, pp. 177183, 2014.

[4] Ahmed, Syed Hassan, Safdar H. Bouk, Nadeem Javaid, and Iwao Sasase. "RF propagation analysis of MICAz Mote's antenna with ground effect," In Multitopic Conference (INMIC), 2012 15th International, pp. 270-274. IEEE, 2012.

[5] C. Caione, D. Brunelli, and L. Benini, "Distributed compressive sampling for lifetime optimization in dense wireless sensor networks", IEEE Trans. Ind. Informat., vol. 8, no. 1, pp. 3040, Feb. 2012.

[6] Madhu Patil, Chirag Sharma, "Energy Efficient WSN by Optimizing the Packet Failure in Network", Indonesian Journal of Electrical Engineering and Computer Science, Vol. 7, No. 2, August 2017, pp. 415 425 DOI: 10.11591/ijeecs.v7.i2.pp415-425 
[7] GK Srinivasa Gowda, CV Srikrishna and Kashyap D Dhruve, "Wireless Measurement Scheme for Bandwidth Estimation in Multihop Wireless Adhoc Network", Global Journal of Computer Science and Technology, vol.13, no.5, pp 1-11, April 2013

[8] J. Peng, X. H. Chen, and T. Liu, "A flow-partitioned unequal clustering routing algorithm for wireless sensor networks”, Int. J. Distrib. Sensor Netw. vol. 2014, 12 pp., 2014, Article ID 875268.

[9] S. Rani; S. H. Ahmed; R. Talwar; J. Malhotra, "Can Sensors Collect Big Data? An Energy Efficient Big Data Gathering Algorithm for WSN," in IEEE Transactions on Industrial Informatics, vol.PP, no.99, pp.1-1, 2017.

[10] C. W. Tsai, T. P. Hong and G. N. Shiu, "Metaheuristics for the Lifetime of WSN: A Review," in IEEE Sensors Journal, vol. 16, no. 9, pp. 2812-2831, May1, 2016.

[11] M. Parsapoor and U. Bilstrup, "An Imperialist Competitive Algorithm for Interference-Aware Cluster-Heads Selection in Ad Hoc Networks," 2014 IEEE 28th International Conference on Advanced Information Networking and Applications, Victoria, BC, 2014, pp. 41-48.

[12] C. H. Chen and W. H. Chen, "United-Based Imperialist Competitive Algorithm for Compensatory Neural Fuzzy Systems," in IEEE Transactions on Systems, Man, and Cybernetics: Systems, vol. 46, no. 9, pp. 1180-1189, Sept. 2016.

[13] D. S. Weile and E. Michielssen, "Genetic algorithm optimization applied to electromagnetics: A review," IEEE Trans. Antennas Propag., vol. 45, no. 3, pp. 343-353, 1997.

[14] J. Prasad and T. Souradeep, "Cosmological parameter estimation using particle swarm optimization (PSO)," Phys. Rev. D, vol. 85, no. 12, Art. ID 123008, 2012.

[15] Z. Xu, L. Chen, C. Chen and X. Guan, "Joint Clustering and Routing Design for Reliable and Efficient Data Collection in Large-Scale Wireless Sensor Networks," in IEEE Internet of Things Journal, vol. 3, no. 4, pp. 520-532, Aug. 2016.

[16] H. K. Deva Sarma, R. Mall and A. Kar, "E2R2: Energy-Efficient and Reliable Routing for Mobile Wireless Sensor Networks," in IEEE Systems Journal, vol. 10, no. 2, pp. 604-616, June 2016.

[17] P. Nayak and B. Vathasavai, "Energy Efficient Clustering Algorithm for Multi-Hop Wireless Sensor Network Using Type-2 Fuzzy Logic," in IEEE Sensors Journal, vol. 17, no. 14, pp. 4492-4499, July15, 152017.

[18] P. Nayak and A. Devulapalli, "A Fuzzy Logic-Based Clustering Algorithm for WSN to Extend the Network Lifetime," in IEEE Sensors Journal, vol. 16, no. 1, pp. 137-144, Jan.1, 2016.

[19] Mohammed Ali Hussain," Energy Efficient Intrusion Detection Scheme with Clustering for Wireless Sensor Networks", TELKOMNIKA Indonesian Journal of Electrical Engineering, Vol. 15, No. 1, July 2015, pp. 128 141. DOI: 10.11591/telkomnika.v15i1.8072,

[20] W.B. Heinzelman, A.P. Chandrakasan, and H. Balakrishnan, "An application-specific protocol architecture for wireless microsensor networks," IEEE Transactions on Wireless Communications, 1(4):660 -670, 2002. (Lifetime).

[21] J. S. Leu, T. H. Chiang, M. C. Yu and K. W. Su, "Energy Efficient Clustering Scheme for Prolonging the Lifetime of Wireless Sensor Network With Isolated Nodes," in IEEE Communications Letters, vol. 19, no. 2, pp. 259-262, Feb. 2015.

[22] P. Nayak and A. Devulapalli, "A Fuzzy Logic-Based Clustering Algorithm for WSN to Extend the Network Lifetime," in IEEE Sensors Journal, vol. 16, no. 1, pp. 137-144, Jan.1, 2016.

[23] H. K. Deva Sarma, R. Mall and A. Kar, "E2R2: Energy-Efficient and Reliable Routing for Mobile Wireless Sensor Networks," in IEEE Systems Journal, vol. 10, no. 2, pp. 604-616, June 2016.

[24] Kashaf, A., Javaid, N., Khan, Z., \& Khan, I., "TSEP: Threshold-sensitive stable election protocol for WSNs," In Conference on Frontiers of information technology, pp. 164-168, 2012.

[25] Mohamed Elhoseny, Ahmed Farouk, Nanrun Zhou, Ming-Ming Wang, Soliman Abdalla, Josep Batle, "Dynamic Multi-hop Clustering in a Wireless Sensor Network: Performance Improvement", Wireless Personal Communications, Springer US, 95(4), Pags 3733-3753 (DOI 